PRENATAL DIAGNOSIS AND POSTNATAL MANAGEIMENT OF THE COMPLETE MOLAR PREGNANCY WITH COEXISTING NORMAL FETUS
KEY WORDS:Complete

mole/free b-human chorionic gonadotrophin/gestational trophoblastic disease/ molar pregnancy
Dr .A .Nagarani* Dr. Dantam

\section{Hymavathi Devi} \section{INTRODUCTION}

Hydatidiform mole with a coexisting fetus is an extremely rare phenomenon; the incidence of such an occurrence ranges from 1 in 10000 to 1 in 100000 gestations(Cunningham et al., 1997). There were two possible conditions: a partial mole with an abnormaltriploid fetus, and a complete mole combined with a normal fetus and placenta. Most cases suffer severe complications, such as pre-eclampsia, abortion and preterm delivery, or termination immediately after the diagnosis.

Delivery of a viable term normal infant from this combination is even rarer. In this report, not only the grounds for the prenatal diagnosis, but also the basis for continuing the pregnancy, and prenatal care wil be discussed. Furthermore, the strong possibility of development of post-molar trophoblastic disease should be considered, especially because of the initial presence of significantly elevated free b-HCG and Complete molar pregnancy. Postpartum management will also be described.

\section{CASE REPORT}

The patient was a 35 year old, gravida 3, para 2, live 2, woman, who presented at 18 weeks gestation for routine obstetric evaluation. Gestation was corresponding to the period of amenorrhea. There were no specific past and family histories. Prenatal Down's syndrome screening was done by checking free ß-human chorionic gonadotrophin (HCG) and $\alpha$-fetal protein(AFP), which revealed elevated free b-HCG[520 $\mathrm{ng} / \mathrm{ml}]$ and normal range of AFP $[52.36 \mathrm{ng} / \mathrm{ml}]$.' $^{\prime}$

At 20 weeks gestation, physical examination revealed a symphysio fundal height of $20 \mathrm{~cm}$, Which is compatible with the gestational age. Ultrasound revealed a live fetus with measurements consistent with a normal 20 weeks of gestation. The amount of amniotic fluid was normal and there was no evidence of growth retardation or fetal anomalies. However, a well-defined and separate multiple cystic, snowstorm like mass was found in the lower segment of uterus, connecting with the normal placenta, thus Coexisting complete hydatidiform mole was strongly suspected. The elevated free $\mathrm{b}-\mathrm{HCG}$ concentration and the risk of possible fetal malformation were considered.

Amniocentesis was done. Karyotyping revealed normal $46, \mathrm{XY}$. The risks of possible fetal malformation or subsequent malignant transformation of the molar pregnancy were explained to the patient. The patient still chose to continue the pregnancy. In the next follow-up, the multicystic mass became more prominent, but without blood flow on Doppler ultrasound and the fetus had stable development without growth retardation. The patient did not present with any complications. corresponding to the period of amenorrhea. At 38 weeks she underwent a elective Caesareansection in view of cephalopelvic disproportion and previous Caesarean section. A male baby with normal appearance, weighing 3100 $g$ and Apgar score of 8 and 10 at 1 and 5 min respectively, was delivered.

The placenta and separate grape-like mass were removed manually. The placenta was roughly disc-shaped with a centrally attached, three vessel umbilical cord. Approximately one-third of the maternal surface of the placenta had the appearance of a classic hydatidi form mole. Histological examination of the grape- like mass showed layers of degenerated, attenuated or hyperplastic sheets of trophoblasts with mild to moderate atypia, the cores of he villi showed 'cistern" formation and vessels were absent, which is compatible with the complete hydatidiform mole. During the postpartum period, the patient receivedregular follow-up of $\beta$-HCG.Serumb-HCG titre had the normal regression curve during the first month after the birth. But during the following month a rising tendency was noted (Figure l). A chest X-ray and Doppler ultrasound examination of the uterus did not reveal any abnormality.

Therefore, the patient received four courses of methotrexate ( $1 \mathrm{mg} / \mathrm{kg}$ every other day combined with folinic acid every other day for 1 week each course). Serum b-HCG titre fell promptly after the first methotrexate course, but then fell slowly and plateaued in the following three methotrexate courses (Figure 1). At this time, chest and abdominal computer tomography and Doppler ultrasound examination of uterus still showed no significant findings. Therefore, the chemotherapy was changed to actinomycin $\mathrm{D}$ treatment $(0.45 \mathrm{mg} / \mathrm{kg}$ for 5 days every course) for three courses, after which serum b-HCG fell to a normal level and has remained so, and the baby was well developed at all visits. At present the patient is well and still has regular follow-up of b-HCG.

\section{DISCUSSION}

Hydatidiform mole can be separated into two entities with respect to cytogenetics, histopathology and morphology: firstly, complete, classical mole has diploid karyotype, no embryo and amnion, and uniform changes of the placental villi and trophoblasts; secondly, partial mole usually has a triploid karyotype, the presence of ascertainable embryo, umbilical cord or an amniotic membrane, and only focal changes of placental villi and trophoblasts (Szulman and Surti, 1978a,b).

Therefore, hydatidiform mole with a coexisting fetus can be established by the partial mole syndrome or by a twin pregnancy where the other conceptus has degenerated into a mole. In our present case, the fetal karyotype is $46, \mathrm{XY}$ and the histopathological and morphological findings of a normal placenta and separated molar tissues with marked hydrophic avascular villi support thetheoretical explanation that it resulted from a twin dizygotic pregnancy in which one twin had developed normally and the other had degenerated into a complete mole. Prenatal diagnosis of coexistent mole and fetus can depend upon the clinical symptoms and signs, physical examination, sonographic findings, and abnormal biochemical data. Clinically, the patient may present with hyperemesis, hyperthyroidism, vaginal spotting or even heavy bleeding, pregnancy-induced hypertension and larger-than-gestational age uterus. However, these conditions are not always present, as none of these symptoms and signs were seen in the present case. Ultrasound examination usually provides a reliable means for the diagnosis of molar pregnancy. The typical ultrasonographic findings of a molar pregnancy consist of a complex cystic pattern with a 'snowstorm' appearance. But it should be differentiated from a missed abortion with its degenerated gestational sac, especially during the early pregnancy, or even a partially 
necrotic leiomyoma which can produce a similar appearance (Sauerbree et al., 1980).

The difference between a partial and complete mole cannot be firmly established by ultrasound because they both present with the same vesicular pattern.

However, in our case, the ultrasound clearly revealed not only a normal appearing fetus, but also a normal placenta connecting with the sharply defined molar tissues. Since the normal placenta separate from molar tissues can be well defined, a complete mole pregnancy with a concurrent fetus can be diagnosed prenatally.

It is important to distinguish between a complete and partial mole when a fetus coexists, because it has been reported that a complete mole has a $20 \%$ tendency to become an invasive mole or even a choriocarcinoma, while the risk was far less for partial moles (Callen, 1988).

The b- HCG titre is generally higher than is seen in non-molar gestations. Free b-HCG is often used for prenatal screening of Down'-syndrome.

Khazaeli et al. (1989) suggested that the excessive production of free b-HCG may identify gestational trophoblastic disease in patients during pregnancy and also identifies patients with a high risk of gestational trophoblastic disease.

However, a relationship between free $\beta$-HCG and coexisting molar pregnancy has not been reported. In the present of pregnancies with mole and fetus in which there case, the patient had an extremely high of concentration of free $\beta-\mathrm{HCG}$ which not only confirmed the sonographic diagnosis of combined molar pregnancy, but also presented the possibility of later developing gestational trophoblastic disease, in addition to the risk from the complete mole and term pregnancy. Therefore, the use of ultrasound, augmented by the significantly elevated $\beta$-HCG value, especially free $\beta$ HCG, and clinical symptoms and signs, has facilitated the accurate prenatal diagnosis of coexistent mole and fetus.

The outcome for a normal pregnancy with a coexisting complete mole is poor, with approximately a $25 \%$ chance of achieving a live birth. There is an increased risk of early fetal loss $(40 \%)$ and premature delivery $(36 \%)$.The incidence of pre- eclampsia is variable, with rates as high as $20 \%$ reported. However, in the large UK series, the incidence was only $4 \%$ and there were no maternal deaths.(sebire NJ,2002;Wee $\mathrm{L}, 2005)$ As a result, management of molar pregnancy with coexisting fetus remains controversial.

In the present case, the decision of the patient to continue the pregnancy was accepted, not only because of normal karyotype, appearance and good fetal development, but also because no significant symptoms and signs presented. Although we demonstrate that a complete molar pregnancy with a normal concurrent fetus can permit the normal development of the fetus until term with a good fetal and maternal outcome, we cannot ignore the high risk of developing persistent gestational trophoblastic disease later, especially with the presence of a Significantly high concentration of free $\beta$-HCG and complete mole. Therefore, proper prenatal consultation should consider both the outcome and the possible complications of such a pregnancy. Vejerslev (1991) reviewed 113 reports of pregnancies with mole and fetus and fetus in which there appeared to be no major malformations or cytogenetic abnormalities. Of these, 87 had intended to continue the pregnancy with or without knowledge of accompanying th mole; in fact, 52 pregnancies (59.8\%) proceeded to the $28 \mathrm{week}$, and a risk for either substantial bleeding or preeclamptic symptoms developed in $\sim 30 \%$.

In $15 \%$ the degree of severity required termination on clinical grounds irrespective of fetal development. Before week 28 there is essentially no hope for a surviving child and the chance for continuation of the pregnancy beyond this point is $60 \%$. Of pregnancies which continue beyond the 28 th week, a surviving child may be expected in $\sim 70 \%$ of pregnancies, the risk for intrauterine or neonatal death being $\sim 30 \%$. On the other hand, persistent trophoblastic disease was reported in $19.2 \%$ of pregnancies interrupted at diagnosis, as well as in $9.1 \%$ of those who had continued (Vejerslev, 1991). In addition, no reports demonstrate that if pregnancy is kept to term, it wil increase the incidence of invasive mole or choriocarcinoma. Nwosuetal. (1995) even demonstrated a case in which there was no sign of recurrence or trophoblastic disease developing in a patient after delivery of coexisting molar pregnancy and a living normal baby at term.

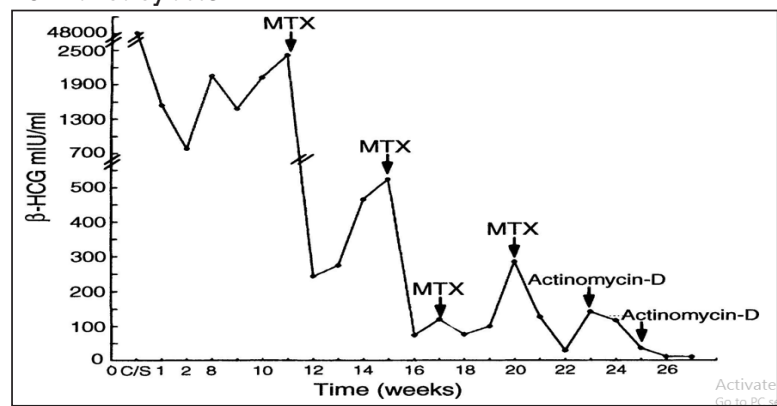

Figure 1. The Changes Of Serum B-human Chorionic Gonadotrophin (hcg) AfterDelivery AndDuring Chemotherapy.

Some reports showed that chemotherapy or hysterectomy were required later, even when termination of the pregnancy during early gestation was required treatment in the condition (Fisher et al., 1982; Teng and Ballon, 1984; Garcia-Aguayo and Irles, 1988). In our case, the postpartum chemotherapy was fairly non-aggressive. It may be better to initiate More aggressive prophylactic chemotherapy immediately after termination of coexisting complete and molar pregnancy.

\section{CONCLUSION}

pragnancy with a coexisting fetus, in addition to the chromosomal evaluation and detailed Ultrasound examination to exclude fetal abnormality before the decision to postpone intervention, prenatal consultation should include complete discussion of maternal and fetal risks , especially the possible requirement of chemotherapy or even hysterectomy . It is also necessary to have close follow-up and evaluation of the condition of the patient during the prenatal and postnatal period.

\section{REFERENCES}

1. Callen, P.W. (1988) Ultrasound evaluation of gestational trophoblastic disease.In Callen, P.W. (ed.), Ultrasonography in Obstetrics and Gynecology. 2nd edn.W.B.Saunders, Philadelphia,PA, p. 416.

2. Cunningham, M.E., Walls, W.L. and Burke, M.F. (1977) Gray scale ultra sonography in the diagnosis of hydatidiform mole with a coexisting fetus. Br. J. Obstet. Gynecol., 84, 73-75.

3. Fisher,R.A.,Sheppard,D.M. and Lawler,S.D. (1982)Twin pregnancy with complete hydatidiform mole $(46, \mathrm{XX})$ and fetus $(46, \mathrm{XY})$ : genetic origin provided by analysis of chromosome polymorphisms.Br.Med.J.284,1218-1220.

4. Garcia-Aguayo, J.F. and Irles, M.A.M. (1988) Evolution of diamniotic dichorionic pregnancy into complete hydatidiform mole and normal fetus. J. Clin. Ultrasound, 20,604-607.

5. Sebire NJ, Foskett M, Paradinas FJ, Fisher RA, Francis RJ, Short D, et al. Outcome of twin pregnancies with complete hydatidi- form mole and healthy co-twin. lancet 2002;359:2165-6.

6. Wee $\mathrm{L}$, Jauniaux E. Prenatal diagnosis and management of twin pregnancie complicated by a co-existing molar pregnancy. Prenat Diagn2005;25:772-6.

7. Khazaeli,M.B.,Buchina,E.S.,Pattillo, R.A. et al. (1989) Radioimmunoassay of free b-subunit of human chorionic gonadotropin in diagnosis of high risk and lowrisk gestational trophoblastic disease.Am.J. Obstet. Gynecol., 160,444-449.

8. Nwosu, E.C. Ferriman, E., McCormack, M.J. et al. (1995) Partial hydatidiform mole and hypertension associated with a live fetus-variable presentation in two cases. Hum. Reprod., 10,2459-2462.

9. Sauerbree, E.E., Shia, S. and Fayle, B. (1980) Coexistent hydatidiform mole and a live fetus in the second trimester. Radiology, 135,415-417.

10. Szulman, A.E. and Surti, U. (1978a) The syndromes of hydatidiform mole. Cytogenetic and morphologic correlations.Am.J.Obstet. Gynecol.,131,665-671.

11. Szulman, A.E. and Surti, U. (1978b) The syndromes of hydatidiform mole. Morphologic evolution ofthecomplete andpartial mole. Am. J. Obstet. Gynecol., 132,20-27.

12. Teng, N.N.H. and Ballon, S.C. (1984) Partial hydatidiform mole with diploid karyotype:report of three cases. Am.J. Obstet. Gynecol., 150,961-964

13. Vejerslev, L.O. (1991) Clinical management and diagnostic possibilities in hydatidiform mole with coexistent fetus. Obstet. Gynecol. Surv., 46,577-588. 